\title{
Nontrivial Solution for the Fractional $p$-Laplacian Equations via Perturbation Methods
}

\author{
Huxiao Luo, ${ }^{1}$ Shengjun $\mathrm{Li}^{1,2}$ and Xianhua Tang ${ }^{1}$ \\ ${ }^{1}$ School of Mathematics and Statistics, Central South University, Changsha, Hunan 410083, China \\ ${ }^{2}$ College of Information Sciences and Technology, Hainan University, Haikou 570228, China
}

Correspondence should be addressed to Shengjun Li; shjli626@126.com

Received 4 August 2017; Accepted 2 November 2017; Published 21 November 2017

Academic Editor: Jacopo Bellazzini

Copyright (C) 2017 Huxiao Luo et al. This is an open access article distributed under the Creative Commons Attribution License, which permits unrestricted use, distribution, and reproduction in any medium, provided the original work is properly cited.

We study the existence of nontrivial solution of the following equation without compactness: $(-\Delta)_{p}^{\alpha} u+|u|^{p-2} u=f(x, u), x \in \mathbb{R}^{N}$, where $N, p \geq 2, \alpha \in(0,1),(-\Delta)_{p}^{\alpha}$ is the fractional $p$-Laplacian, and the subcritical $p$-superlinear term $f \in C\left(\mathbb{R}^{N} \times \mathbb{R}\right)$ is 1-periodic in $x_{i}$ for $i=1,2, \ldots, N$. Our main difficulty is that the weak limit of (PS) sequence is not always the weak solution of fractional $p$-Laplacian type equation. To overcome this difficulty, by adding coercive potential term and using mountain pass theorem, we get the weak solution $u_{\lambda}$ of perturbation equations. And we prove that $u_{\lambda} \rightarrow u$ as $\lambda \rightarrow 0$. Finally, by using vanishing lemma and periodic condition, we get that $u$ is a nontrivial solution of fractional $p$-Laplacian equation.

\section{Introduction}

This article is concerned with the fractional $p$-Laplacian equations

$$
(-\Delta)_{p}^{\alpha} u+|u|^{p-2} u=f(x, u), \quad x \in \mathbb{R}^{N},
$$

where $N, p \geq 2, \alpha \in(0,1)$, and $f$ satisfies the following conditions.

$\left(f_{1}\right) f \in C\left(\mathbb{R}^{N} \times \mathbb{R}\right), f$ is 1-periodic in $x_{i}$ for $i=1,2, \ldots, N$, and

$$
\begin{gathered}
\lim _{|t| \rightarrow \infty} \frac{f(x, t)}{|t|^{q-1}}=0, \\
\lim _{|t| \rightarrow \infty} \frac{f(x, t) t}{|t|^{p}}=+\infty
\end{gathered}
$$

uniformly in $x \in \mathbb{R}^{N}$ for some $q \in\left(p, p_{\alpha}^{*}\right)$, where $p_{\alpha}^{*}=N p /(N-\alpha p)$;

$\left(f_{2}\right) f(x, t)=o\left(|t|^{p-2} t\right)$ as $|t| \rightarrow 0$ uniformly for $x \in \mathbb{R}^{N}$; $\left(f_{3}\right) \widetilde{F}(x, u):=(1 / p) f(x, u) u-F(x, u)>0$ if $u \neq 0$, and there exists $c_{0} \geq 0$ and $\sigma>\max \{N / p \alpha, 1\}$ such that

$$
|f(x, u)|^{\sigma} \leq c_{0}|u|^{\sigma(p-1)} \widetilde{F}(x, u), \quad \forall x \in \mathbb{R}^{N},|u| \geq r_{0},
$$

where $F(x, t)=\int_{0}^{t} f(x, s) d s$.

The fractional $p$-Laplacian is defined on smooth functions by

$$
\begin{array}{r}
(-\Delta)_{p}^{\alpha} u(x) \\
=2 \lim _{\epsilon \rightarrow 0} \int_{\mathbb{R}^{N} \backslash B_{\epsilon}(x)} \frac{|u(x)-u(y)|^{p-2}(u(x)-u(y))}{|x-y|^{N+\alpha p}} d y, \\
x \in \mathbb{R}^{N} .
\end{array}
$$

This definition is consistent, up to a normalization constant depending on $N$ and $\alpha$, with the usual definition of the linear fractional Laplacian operator $(-\Delta)^{\alpha}$ when $p=2$. There is, currently, a rapidly growing literature on problems involving these nonlocal operators. This type of problem arises in many different applications, such as continuum mechanics, phase transition phenomena, population dynamics, and 
game theory, as they are the typical outcome of stochastically stabilization of Lévy processes; see [1-9] and the references therein. The literature on nonlocal operators and their applications is very interesting and quite large; we refer the interested reader to $[4,10-21]$ and the references therein. For the basic properties of fractional Sobolev spaces, we refer the interested reader to $[22,23]$.

The main purpose of this paper is to consider the existence of nontrivial solutions for equation (1). Our main difficulty is that the weak limit of (PS) sequence is not always the weak solution of (1). To overcome this problem, we apply the perturbation method [22, 24-26]. First, we consider the perturbation equation by adding coercive potential term

$$
\begin{aligned}
(-\Delta)_{p}^{\alpha} u+\lambda V(x)|u|^{p-2} u+|u|^{p-2} u=f(x, u), & \\
& x \in \mathbb{R}^{N},
\end{aligned}
$$

where $\lambda \in(0,1]$ is a parameter and $V(x)$ satisfies the following conditions:

$$
\begin{aligned}
& \left(V_{1}\right) V \in C\left(\mathbb{R}^{N}\right), \inf _{\mathbb{R}^{N}} V(x) \geq V_{0}>0 . \\
& \left(V_{2}\right) \text { meas }\left\{x \in \mathbb{R}^{N} \mid V(x) \leq M\right\}<+\infty, \forall M>0 .
\end{aligned}
$$

And we prove that the energy functional of (5) has the geometry of the mountain pass theorem that it satisfies the Cerami condition and finally that the obtained solutions $\left\{u_{\lambda}\right\}$ have the uniform bounds. Finally, we verify that $u_{\lambda} \rightarrow u$ as $\lambda \rightarrow 0$ and $u$ is the nontrivial solution of (1).

Now, we give the main result of this article.

Theorem 1. Suppose that $\left(f_{1}\right)-\left(f_{3}\right)$ hold. Then (1) possesses at least a nontrivial solution.

Remark 2. In order to get our result, there are mainly three difficulties.

(i) The working space has not compactness.

(ii) The classical AR condition for the nonlinearity is not satisfied.

(iii) If $\left\{u_{n}\right\}$ is a Palais Smale sequence of $\Phi$ (see Section 2) and $u_{n}$ converges weakly to $u_{0}$, one can not obtain that $u_{0}$ is a weak solution of the fractional $p$-Laplacian type equation (1).

Notation 1. In this paper we make use of the following notation:

(i) $\|\cdot\|_{p}$ is the usual norm of the space $L^{p}\left(\mathbb{R}^{N}\right)$.

(ii) $c, C$ and $c_{i}, C_{i}$ denote positive (possibly different) constants.

(iii) We denote the weak convergence in $X$ and its $X^{*}$ by " $\rightarrow$ " and the strong convergence by " $\rightarrow$ ".

(iv) $o$ (1) denote being infinitely small (possibly different) when $n \rightarrow \infty$.

\section{Variational Framework}

Before stating this section, we define the Gagliardo seminorm by

$$
[u]_{\alpha, p}=\left(\int_{2 \mathbb{R}^{N}} \frac{|u(x)-u(y)|^{p}}{|x-y|^{N+\alpha p}} d x d y\right)^{1 / p},
$$

where $u: \mathbb{R}^{N} \rightarrow \mathbb{R}$ is a measurable function. On one hand, we define fractional Sobolev space by

$$
\begin{aligned}
W^{\alpha, p} & \left(\mathbb{R}^{N}\right) \\
\quad & \left\{u \in L^{p}\left(\mathbb{R}^{N}\right): u \text { is measurable, }[u]_{\alpha, p}<\infty\right\}
\end{aligned}
$$

endowed with the norm

$$
\|u\|_{\alpha, p}=\left([u]_{\alpha, p}^{p}+\|u\|_{p}^{p}\right)^{1 / p}
$$

where

$$
\|u\|_{p}=\left(\int_{\mathbb{R}^{N}}|u(x)|^{p} d x\right)^{1 / p} .
$$

Moreover, (1) is variational and its solutions are the critical points of the functional defined in $W^{\alpha, p}\left(\mathbb{R}^{N}\right)$ by

$$
\begin{aligned}
& \Phi(u)=\frac{1}{p}[u]_{\alpha, p}^{p}+\frac{1}{p}\|u\|_{p}^{p}-\int_{\mathbb{R}^{N}} F(x, u) d x, \\
& \forall u \in W^{\alpha, p} .
\end{aligned}
$$

From $\left(f_{1}\right)$, it is easy to check that $\Phi$ is well defined on $W^{\alpha, p}\left(\mathbb{R}^{N}\right)$ and $\Phi \in C^{1}\left(W^{\alpha, p}\left(\mathbb{R}^{N}\right), \mathbb{R}\right)$, and

$$
\begin{aligned}
& \left\langle\Phi^{\prime}(u), v\right\rangle \\
& =\int_{2 \mathbb{R}^{N}} \frac{|u(x)-u(y)|^{p-2}(u(x)-u(y))(v(x)-v(y))}{|x-y|^{N+\alpha p}} d x d y \\
& \quad+\int_{\mathbb{R}^{N}}|u|^{p-2} u v d x-\int_{\mathbb{R}^{N}} f(x, u) v d x .
\end{aligned}
$$
space

On the other hand, we consider the fractional Sobolev

$$
X^{\alpha}:=\left\{u \in W^{\alpha, p}: \int_{\mathbb{R}^{N}} V(x)|u|^{p} d x<\infty\right\}
$$

endowed with the norm

$$
\|u\|:=\|u\|_{X^{\alpha}}=\left([u]_{\alpha, p}^{p}+\int_{\mathbb{R}^{N}} V(x)|u|^{p} d x\right)^{1 / p} .
$$

We also need the following inner norm:

$$
\|u\|_{\lambda}=\left([u]_{\alpha, p}^{p}+\lambda \int_{\mathbb{R}^{N}} V(x)|u|^{p} d x\right)^{1 / p}
$$

and let $X_{\lambda}^{\alpha}=\left(X^{\alpha},\|\cdot\|_{\lambda}\right)$. Obviously, we have

$$
\lambda\|u\|^{p} \leq\|u\|_{\lambda}^{p} \leq\|u\|^{p}
$$

the two norms $\|\cdot\|$ and $\|\cdot\|_{\lambda}$ are equivalent. Next, the following lemma discusses the continuous and compact embedding for $X^{\alpha} \hookrightarrow L^{q}\left(\mathbb{R}^{N}\right)$ for all $q \in\left[p, p_{\alpha}^{*}\right]$. For the proof of the lemma, it was proved in [27] in the case $p=2$. For the general case, the proof is similar. We give it here for readers convenience. 
Lemma 3. Assume that $\left(V_{1}\right)$ and $\left(V_{2}\right)$ hold. Then $X^{\alpha}$ is continuously embedded in $L^{s}\left(\mathbb{R}^{N}\right)$ for all $s \in\left[p, p_{\alpha}^{*}\right]$. Moreover, $X^{\alpha}$ can be compactly embedded into $L^{s}\left(\mathbb{R}^{N}\right)$ for all $s \in\left[p, p_{\alpha}^{*}\right)$.

Proof. Let $\left\{u_{n}\right\} \subset X^{\alpha}$ be a bounded sequence of $X^{\alpha}$ such that $u_{n} \rightarrow 0$ in $X^{\alpha}$. Then, by Theorem 2.1 in [22], $u_{n} \rightarrow 0$ in $L_{\text {loc }}^{q}\left(\mathbb{R}^{N}\right)$ for $p \leq q<p_{\alpha}^{*}$. We claim that

$$
u_{n} \longrightarrow 0 \quad \text { strongly in } L^{p}\left(\mathbb{R}^{N}\right) .
$$

To prove (16), we only need to show that, for any $\varepsilon>0$, there exists $R>0$ such that

$$
\int_{\mathbb{R}^{N} \backslash B_{R}}\left|u_{n}(x)\right|^{p} d x<\varepsilon .
$$

Set

$$
\begin{aligned}
B_{R} & =\left\{x \in \mathbb{R}^{N}|| x \mid<R\right\}, \\
A(R, M) & =\left\{x \in \mathbb{R}^{N} \backslash B_{R} \mid V(x) \geq M\right\}, \\
B(R, M) & =\left\{x \in \mathbb{R}^{N} \backslash B_{R} \mid V(x)<M\right\} ;
\end{aligned}
$$

then

$$
\begin{aligned}
\int_{A(R, M)}\left|u_{n}(x)\right|^{p} d x & \leq \int_{\mathbb{R}^{N}} \frac{V(x)}{M}\left|u_{n}(x)\right|^{p} d x \\
& \leq \frac{\left\|u_{n}\right\|_{X^{\alpha}}^{p}}{M} .
\end{aligned}
$$

Now choose $\sigma \in\left(1, p_{\alpha}^{*} / p\right)$ such that $1 / \sigma+1 / \sigma^{\prime}=1$; then we have

$$
\begin{aligned}
& \int_{B(R, M)}\left|u_{n}(x)\right|^{p} d x \\
& \quad \leq\left(\int_{B(R, M)}\left|u_{n}(x)\right|^{p \sigma} d x\right)^{1 / \sigma}(\operatorname{meas}(B(R, M)))^{1 / \sigma^{\prime}} \\
& \quad \leq C\left\|u_{n}\right\|_{X^{\alpha}}^{p}(\operatorname{meas}(B(R, M)))^{1 / \sigma^{\prime}} .
\end{aligned}
$$

Since $\left\|u_{n}\right\|_{X^{\alpha}}^{p}$ is bounded and condition $\left(V_{2}\right)$ holds, we may choose $R, M$ large enough such that $\left\|u_{n}\right\|_{X^{\alpha}}^{p} / M$ and meas $(B(R, M))$ are small enough. Hence, $\forall \varepsilon>0$, we have

$$
\begin{aligned}
\int_{\mathbb{R}^{N} \backslash B_{R}}\left|u_{n}(x)\right|^{p} d x= & \int_{A(R, M)}\left|u_{n}(x)\right|^{p} d x \\
& +\int_{B(R, M)}\left|u_{n}(x)\right|^{p} d x<\varepsilon
\end{aligned}
$$

from which (16) follows.

To prove the lemma for general exponent $q$, we use an interpolation argument. Let $u_{n} \rightarrow 0$ in $X^{\alpha}$, we have just proved that $u_{n} \rightarrow 0$ in $L^{p}\left(\mathbb{R}^{N}\right)$. That is,

$$
\int_{\mathbb{R}^{N}}\left|u_{n}(x)\right|^{p} d x \longrightarrow 0
$$

as $n \rightarrow \infty$. Moreover, because the embedding $X^{\alpha} \rightarrow$ $L^{p_{\alpha}^{*}}\left(\mathbb{R}^{N}\right)$ is continuous and $\left\{u_{n}\right\}$ is bounded in $X^{\alpha}$, we also have

$$
\sup _{n} \int_{\mathbb{R}^{N}}\left|u_{n}(x)\right|^{p_{\alpha}^{*}} d x<\infty .
$$

Since $q \in\left(p, p_{\alpha}^{*}\right)$, there is a number $\lambda \in(0,1)$ such that $1 / q=$ $\lambda / p+(1-\lambda) / p_{\alpha}^{*}$. Then by Hölder inequality

$$
\begin{aligned}
\int_{\mathbb{R}^{N}}\left|u_{n}(x)\right|^{q} d x & =\int_{\mathbb{R}^{N}}\left|u_{n}(x)\right|^{\lambda q}\left|u_{n}(x)\right|^{1-\lambda q} d x \\
& \leq\left\|u_{n}\right\|_{p}^{\lambda q}\left\|u_{n}\right\|_{p_{\alpha}^{*}}^{(1-\lambda) q} \longrightarrow 0 .
\end{aligned}
$$

This implies $u_{n} \rightarrow 0$ in $L^{q}\left(\mathbb{R}^{N}\right)$.

From Lemma 3, there exists $\gamma_{s}>0$ such that

$$
\|u\|_{s} \leq \gamma_{s}\|u\|, \quad \forall u \in X^{\alpha},
$$

where $\|u\|_{s}$ denotes the usual norm in $L^{s}\left(\mathbb{R}^{N}\right)$ for all $p \leq s \leq$ $p_{\alpha}^{*}$.

Next, we define the energy functional $\Phi_{\lambda}$ on $X^{\alpha}$ by

$$
\begin{aligned}
\Phi_{\lambda}(u) & =\frac{1}{p}\|u\|_{\lambda}^{p}+\frac{1}{p}\|u\|_{p}^{p}-\int_{\mathbb{R}^{N}} F(x, u) d x \\
& =\Phi(u)+\frac{\lambda}{p} \int_{\mathbb{R}^{N}} V(x) u^{p} d x, \quad \forall u \in X^{\alpha} .
\end{aligned}
$$

We also need the following inner norm:

$$
\|u\|_{\lambda, p}=\left(\|u\|_{\lambda}^{p}+\|u\|_{p}^{p}\right)^{1 / p} ;
$$

by Lemma 3, we have that the norms $\|\cdot\|$ and $\|\cdot\|_{\lambda, p}$ are also equivalent. By $\left(V_{1}\right),\left(V_{2}\right)$, the energy functional $\Phi_{\lambda}$ : $X^{\alpha} \rightarrow \mathbb{R}$ is well defined and of class $C^{1}\left(X^{\alpha}, \mathbb{R}\right)$. Moreover, the derivative of $\Phi_{\lambda}$ is

$$
\begin{aligned}
& \left\langle\Phi_{\lambda}^{\prime}(u), v\right\rangle \\
& =\int_{2 \mathbb{R}^{N}} \frac{|u(x)-u(y)|^{p-2}(u(x)-u(y))(v(x)-v(y))}{|x-y|^{N+\alpha p}} d x d y \\
& \quad+\lambda \int_{\mathbb{R}^{N}} V(x)|u|^{p-2} u v d x+\int_{\mathbb{R}^{N}}|u|^{p-2} u v d x \\
& \quad-\int_{\mathbb{R}^{N}} f(x, u) v d x \\
& =\left\langle\Phi^{\prime}(u), v\right\rangle+\lambda \int_{\mathbb{R}^{N}} V(x)|u|^{p-2} u v d x
\end{aligned}
$$

for all $u, v \in X^{\alpha}$.

In what follows, we give the vanishing lemma which is introduced by Lion.

Lemma 4 (see [28]). Assume $\left\{u_{k}\right\}$ is a bounded sequence in $W^{\alpha, p}$ which satisfies

$$
\lim _{k \rightarrow+\infty} \sup _{y \in R^{N}} \int_{B_{R}(y)}\left|u_{k}(x)\right|^{p} d x=0
$$

for some $R>0$. Then

$$
u_{k} \longrightarrow 0 \quad \text { in } L^{q}, \forall p<q<\frac{N p}{N-\alpha p} .
$$




\section{Proofs of the Main Result}

The proof of Theorem 1 is divided into several lemmas. We show that the functional $\Phi_{\lambda}$ has the geometry of the mountain pass theorem that it satisfies the Cerami condition and finally that the obtained solutions have the uniform bounds.

Lemma 5. Suppose that $\left(V_{1}\right),\left(V_{2}\right),\left(f_{1}\right)-\left(f_{3}\right)$ are satisfied. Then there exists $\rho>0, \eta>0$, such that $\inf \left\{\Phi_{\lambda}(u) \mid u \in\right.$ $\left.X^{\alpha},\|u\|=\rho\right\}>\eta$ for fixed $\lambda \in(0,1]$, where $\rho$ and $\eta$ are independent of $\lambda$.

Proof. For any $\varepsilon>0$, it follows from $\left(f_{1}\right)$ and $\left(f_{2}\right)$ that there exists $C_{\varepsilon}$ such that

$$
|f(x, s)| \leq \varepsilon|s|^{p-1}+C_{\varepsilon}|s|^{q-1}, \quad s \in \mathbb{R}, \quad x \in \mathbb{R}^{N},
$$

where $p<q<p_{\alpha}^{*}$, and then

$$
|F(x, s)| \leq \frac{\varepsilon}{p}|s|^{p}+\frac{C_{\varepsilon}}{q}|s|^{q}, \quad s \in \mathbb{R}, x \in \mathbb{R}^{N} .
$$

For $\rho>0$, let

$$
\Sigma_{\rho}=\left\{u \in X^{\alpha} \mid\|u\| \leq \rho\right\} .
$$

So, from the Sobolev inequality, one has

$$
\begin{aligned}
\left|\int_{\mathbb{R}^{N}} F(x, u) d x\right| & \leq \frac{\varepsilon}{p} \int_{\mathbb{R}^{N}}|u|^{p} d x+\frac{C_{\varepsilon}}{q} \int_{\mathbb{R}^{N}}|u|^{q} d x \\
& \leq \frac{\gamma_{p} \varepsilon}{p}\|u\|^{p}+\frac{\gamma_{q} C_{\varepsilon}}{q}\|u\|^{q} .
\end{aligned}
$$

So one has, for $u \in \partial \Sigma_{\rho}$,

$$
\begin{aligned}
\Phi_{\lambda}(u)= & \frac{1}{p}[u]_{\alpha, p}^{p}+\frac{1}{p}\|u\|_{p}^{p}+\frac{\lambda}{p} \int_{\mathbb{R}^{N}} V(x)|u|^{p} d x \\
& -\int_{\mathbb{R}^{N}} F(x, u) d x \\
\geq & \frac{\lambda}{p}[u]_{\alpha, p}^{p}+\frac{\lambda}{p} \int_{\mathbb{R}^{N}} V(x)|u|^{p} d x-\frac{\gamma_{p} \varepsilon}{p}\|u\|^{p} \\
& -\frac{\gamma_{q} C_{\varepsilon}}{q}\|u\|^{q}=\frac{\lambda-\gamma_{p} \varepsilon}{p}\|u\|^{p}-\frac{\gamma_{q} C_{\varepsilon}}{q}\|u\|^{q},
\end{aligned}
$$

since $0<\lambda \leq 1$. Hence, by fixing $\varepsilon \in\left(0,1 / \lambda \gamma_{p}\right)$ and letting $\rho>0$ be small enough, it is easy to see that there is $\eta>0$ such that this lemma holds.

Lemma 6. Suppose that $\left(V_{1}\right),\left(V_{2}\right),\left(f_{1}\right)-\left(f_{3}\right)$ are satisfied. then there exists $e \in X^{\alpha}$ with $\|e\|>\rho$ such that $\Phi_{\lambda}(e)<0$ for fixed $\lambda \in(0,1]$, where $\rho$ is given by Lemma 5 .

Proof. Using $\left(f_{1}\right)$, we obtain there exists $T>0$ such that

$$
F(x, t)>\frac{|t|^{p}}{\varepsilon}-\frac{T^{p}}{\varepsilon}, \quad t \in \mathbb{R}, x \in \mathbb{R}^{N} .
$$

Next, for $\varphi \in C_{0}^{\infty}\left(\mathbb{R}^{N}\right)$ we have

$$
\int_{\mathbb{R}^{N}} \frac{F(x, t \varphi)}{|t|^{p}} d x \geq \frac{1}{\varepsilon} \int_{\mathbb{R}^{N}}|\varphi|^{p} d x-\frac{T^{p}}{\varepsilon|t|^{p}} \int_{\operatorname{supp}(\varphi)} d x .
$$

This implies

$$
\lim _{|t| \rightarrow \infty} \int_{\mathbb{R}^{N}} \frac{F(x, t \varphi)}{|t|^{p}} d x \geq \frac{1}{\varepsilon} \int_{\mathbb{R}^{N}}|\varphi|^{p} d x,
$$

for all $\varepsilon>0$. Since $\varepsilon$ is arbitrary, by the above inequality, we get

$$
\lim _{|t| \rightarrow \infty} \int_{\mathbb{R}^{N}} \frac{F(x, t \varphi)}{|t|^{p}} d x=+\infty .
$$

Consequently,

$$
\begin{aligned}
\frac{\Phi_{\lambda}(t \varphi)}{|t|^{p}} & =\frac{1}{p}\|\varphi\|_{\lambda}^{p}+\frac{1}{p}\|\varphi\|_{p}^{p}-\int_{\mathbb{R}^{N}} \frac{F(x, t \varphi)}{|t|^{p}} d x \\
& \leq \frac{1}{p}\|\varphi\|^{p}+\frac{1}{p}\|\varphi\|_{p}^{p}-\int_{\mathbb{R}^{N}} \frac{F(x, t \varphi)}{|t|^{p}} d x
\end{aligned}
$$

$$
\longrightarrow-\infty
$$

as $|t| \rightarrow+\infty$. Hence, let $t_{0}$ be big enough and $e=t_{0} \varphi$; then we have $\Phi_{\lambda}(e)<0$; we complete the proof.

Definition 7. We say that $J$ satisfies Cerami condition in $E$, if, for any sequence $\left\{u_{n}\right\} \subset E$ such that

$$
\begin{aligned}
J\left(u_{n}\right) & \longrightarrow c, \\
\left(1+\left\|u_{n}\right\|_{E}\right) J^{\prime}\left(u_{n}\right) & \longrightarrow 0,
\end{aligned}
$$

as $n \rightarrow \infty$, there exists a convergent subsequence of $\left\{u_{n}\right\}$.

Lemma 8. Suppose that $\left(V_{1}\right),\left(V_{2}\right)$ and $\left(f_{1}\right)-\left(f_{3}\right)$ are satisfied. Then the functional $\Phi_{\lambda}\left(u_{n}\right)$ satisfies Cerami condition.

Proof. Let $\left\{u_{n}\right\}$ be a sequence in $X^{\alpha}$ so that

$$
\begin{aligned}
\Phi_{\lambda}\left(u_{n}\right) & \longrightarrow c_{\lambda}, \\
\left(1+\left\|u_{n}\right\|\right) \Phi_{\lambda}^{\prime}\left(u_{n}\right) & \longrightarrow 0 .
\end{aligned}
$$

We shall prove that $\left\{u_{n}\right\}$ contains a convergent subsequence.

(i) We claim that $\left\{u_{n}\right\}$ is bounded in $X^{\alpha}$. Observe that for $n$ large

$$
c_{\lambda}+1 \geq \Phi_{\lambda}\left(u_{n}\right)-\frac{1}{p} \Phi_{\lambda}^{\prime}\left(u_{n}\right) u_{n}=\int_{\mathbb{R}^{N}} \widetilde{F}\left(x, u_{n}\right) d x .
$$

Arguing indirectly, assume by contradiction that $\left\|u_{n}\right\| \rightarrow \infty$; then $\left\|u_{n}\right\|_{\lambda, p} \rightarrow \infty$. Set $v_{n}=u_{n} /\left\|u_{n}\right\|_{\lambda, p}$; then $\left\|v_{n}\right\|_{\lambda, p}=1$. By Lemma 3, one has $\left\|v_{n}\right\|_{s} \leq \gamma_{s}\left\|v_{n}\right\|_{\lambda, p}=\gamma_{s}$ for $s \in\left[p, p_{\alpha}^{*}\right)$. Observe that from (42) and

$$
\Phi_{\lambda}^{\prime}\left(u_{n}\right) u_{n}=\left\|u_{n}\right\|_{\lambda, p}^{p}\left(1-\int_{\mathbb{R}^{N}} \frac{f\left(x, u_{n}\right) v_{n}}{\left\|u_{n}\right\|_{\lambda, p}^{p-1}} d x\right)
$$


it follows that

$$
\int_{\mathbb{R}^{N}} \frac{f\left(x, u_{n}\right) v_{n}}{\left\|u_{n}\right\|_{\lambda, p}^{p-1}} d x=\int_{\mathbb{R}^{N}} \frac{f\left(x, u_{n}\right)}{u_{n}^{p-1}} v_{n}^{p} d x \longrightarrow 1 .
$$

Set for $r \geq 0$

$$
h(r):=\inf \left\{\widetilde{F}(x, u) \mid x \in \mathbb{R}^{N}, u \in \mathbb{R} \text { with }|u| \geq r\right\} .
$$

By $\left(f_{3}\right), h(r)>0$ for all $r>0$, and $h(r) \rightarrow+\infty$ as $r \rightarrow+\infty$. For $0 \leq a<b$ let

$$
\begin{aligned}
& \Omega_{n}(a, b)=\left\{x \in \mathbb{R}^{N}|a \leq| u_{n}(x) \mid<b\right\} \\
& c_{a}^{b}:=\inf \left\{\frac{\widetilde{F}(x, u)}{u^{p}} \mid x \in \mathbb{R}^{N}, u \in \mathbb{R} \text { with } a \leq|u|\right. \\
& \quad \leq b\} .
\end{aligned}
$$

Since $\widetilde{F}(x, u)>0$ if $u \neq 0$, one has $c_{a}^{b}>0$ and

$$
\widetilde{F}\left(x, u_{n}(x)\right) \geq c_{a}^{b}\left|u_{n}(x)\right|^{p}, \quad \forall x \in \Omega_{n}(a, b) .
$$

It follows from (43) that

$$
\begin{aligned}
c_{\lambda}+1 \geq & \int_{\Omega_{n}(0, a)} \tilde{F}\left(x, u_{n}\right) d x+\int_{\Omega_{n}(a, b)} \tilde{F}\left(x, u_{n}\right) d x \\
& +\int_{\Omega_{n}(b, \infty)} \widetilde{F}\left(x, u_{n}\right) d x \\
\geq & \int_{\Omega_{n}(0, a)} \widetilde{F}\left(x, u_{n}\right) d x+c_{a}^{b} \int_{\Omega_{n}(a, b)}\left|u_{n}\right|^{p} d x \\
& +h(b)\left|\Omega_{n}(b,+\infty)\right| .
\end{aligned}
$$

Invoking $\left(f_{3}\right)$, set $\tau:=p \sigma /(\sigma-1)$ and $\sigma^{*}=\sigma /(\sigma-1)=\tau / p$. Since $\sigma>\max \{1, N / p \alpha\}$ one sees $\tau \in\left(p, p_{\alpha}^{*}\right)$. Fix arbitrarily $s \in\left(\tau, p_{\alpha}^{*}\right)$. Using (49),

$$
\left|\Omega_{n}(b,+\infty)\right| \leq \frac{c+1}{h(b)} \longrightarrow 0
$$

as $b \rightarrow+\infty$ uniformly in $n$, which implies by Hölder inequality that

$$
\begin{aligned}
& \int_{\Omega_{n}(b,+\infty)}\left|v_{n}\right|^{\tau} d x \\
& \leq\left[\int_{\Omega_{n}(b,+\infty)}\left|v_{n}\right|^{s} d x\right]^{\tau / s}\left|\Omega_{n}(b,+\infty)\right|^{1-\tau / s} \\
& \leq \gamma_{s}^{\tau}\left|\Omega_{n}(b,+\infty)\right|^{1-\tau / s} \longrightarrow 0
\end{aligned}
$$

as $b \rightarrow+\infty$ uniformly in $n$. Using (49) again, for any fix $0<$ $a<b$,

$$
\begin{aligned}
\int_{\Omega_{n}(a, b)}\left|v_{n}\right|^{p} d x & =\frac{1}{\left\|u_{n}\right\|_{\lambda, p}^{p}} \int_{\Omega_{n}(a, b)}\left|u_{n}\right|^{p} d x \\
& \leq \frac{c_{\lambda}+1}{c_{a}^{b}\left\|u_{n}\right\|_{\lambda, p}^{p}} \longrightarrow 0
\end{aligned}
$$

as $n \rightarrow+\infty$.
Let $0<\varepsilon<1 / 3$. By $\left(f_{2}\right)$ there is $a_{\varepsilon}>0$ such that $|f(x, u)|<\left(\varepsilon / \gamma_{p}\right)|u|^{p-1}$ for all $|u| \leq a_{\varepsilon}$; consequently,

$$
\begin{aligned}
& \int_{\Omega_{n}\left(0, a_{\varepsilon}\right)} \frac{f\left(x, u_{n}\right)}{u_{n}^{p-1}} v_{n}^{p} d x \leq \int_{\Omega_{n}\left(0, a_{\varepsilon}\right)} \frac{\varepsilon}{\gamma_{p}}\left|v_{n}\right|^{p} d x \\
& \leq \frac{\varepsilon}{\gamma_{p}}\left\|v_{n}\right\|_{p}^{p} \leq \varepsilon
\end{aligned}
$$

for all $n$. By $\left(f_{3}\right),(43),(51)$, and Hölder inequality we can take $b_{\varepsilon} \geq r_{0}$ large so that

$$
\begin{aligned}
& \int_{\Omega_{n}\left(b_{\varepsilon},+\infty\right)} \frac{f\left(x, u_{n}\right)}{u_{n}^{p-1}} v_{n}^{p} d x \\
& \leq\left(\int_{\Omega_{n}\left(b_{\varepsilon},+\infty\right)} \frac{\left|f\left(x, u_{n}\right)\right|^{\sigma}}{\left|u_{n}\right|^{\sigma(p-1)}} d x\right)^{1 / \sigma} \\
& \cdot\left(\int_{\Omega_{n}\left(b_{\varepsilon},+\infty\right)}\left|v_{n}\right|^{\tau} d x\right)^{1 / \sigma^{*}} \\
& \quad \leq\left(\int_{\mathbb{R}^{N}} c_{0} \widetilde{F}\left(x, u_{n}\right) d x\right)^{1 / \sigma}\left(\int_{\mathbb{R}^{N}}\left|v_{n}\right|^{\tau} d x\right)^{(p-1) / \tau} \\
& \cdot\left(\int_{\Omega_{n}\left(b_{\varepsilon},+\infty\right)}\left|v_{n}\right|^{\tau} d x\right)^{1 / \tau}<\varepsilon
\end{aligned}
$$

for all $n$. Note that there is $\gamma=\gamma(\varepsilon)>0$ independent of $n$ such that $\left|f\left(x, u_{n}\right)\right| \leq \gamma\left|u_{n}\right|^{p-1}$ for $x \in \Omega_{n}\left(a_{\varepsilon}, b_{\varepsilon}\right)$. By (52) there is $n_{0}$ such that

$$
\int_{\Omega_{n}\left(a_{\varepsilon}, b_{\varepsilon}\right)} \frac{f\left(x, u_{n}\right)}{u_{n}^{p-1}} v_{n}^{p} d x \leq \gamma \int_{\Omega_{n}\left(a_{\varepsilon}, b_{\varepsilon}\right)} v_{n}^{p} d x<\varepsilon
$$

for all $n \geq n_{0}$. Now the combination of (53), (55), and (62) implies that for $n \geq n_{0}$

$$
\int_{\mathbb{R}^{N}} \frac{f\left(x, u_{n}\right)}{u_{n}^{p-1}} v_{n}^{p} d x<3 \varepsilon<1
$$

which contradicts (45). Hence $\left\{u_{n}\right\}$ is bounded in $X^{\alpha}$.

(ii) By (i), we can conclude that $\left\{u_{n}\right\}$ is bounded in $X^{\alpha}$. Going if necessary to a subsequence, we can assume that $u_{n} \rightarrow u$ in $X^{\alpha}$. From Lemma 3, we have $u_{n} \rightarrow u$ in $L^{s}\left(\mathbb{R}^{N}\right)$ for all $p \leq s<p_{\alpha}^{*}$. By the boundedness of $\left\{u_{n}\right\}$ in $L^{p}\left(\mathbb{R}^{N}\right)$, we have

$$
\Lambda_{1}=\sup _{n} \int_{\mathbb{R}^{N}}\left|u_{n}\right|^{p} d x<\infty
$$

By Hölder inequality and the above inequality we also have

$$
\begin{aligned}
\Lambda_{2} & =\sup _{n} \int_{\mathbb{R}^{N}}\left|u_{n}\right|^{p-1} u d x \\
& \leq \sup _{n}\left(\int_{\mathbb{R}^{N}}\left|u_{n}\right|^{p} d x\right)^{(p-1) / p}\|u\|_{p}<\infty .
\end{aligned}
$$


Similarly,

$$
\Lambda_{3}=\sup _{n} \int_{\mathbb{R}^{N}}|u|^{p-1} u_{n} d x<\infty
$$

By $\left(f_{1}\right),\left(f_{2}\right)$, for $\varepsilon>0$, there exists $C_{\varepsilon}>0$ such that

$$
|f(x, t)| \leq \varepsilon|t|^{p-1}+C_{\varepsilon}|t|^{q-1}, \quad \forall(x, t) \in \mathbb{R}^{N} \times \mathbb{R} .
$$

Then using Hölder inequality we have

$$
\begin{aligned}
& \int_{\mathbb{R}^{N}}\left(f\left(x, u_{n}\right)-f(x, u)\right)\left(u_{n}-u\right) d x \\
& \quad \leq \int_{\mathbb{R}^{N}}\left[\varepsilon\left(\left|u_{n}\right|^{p-1}+|u|^{p-1}\right)\right. \\
& \left.\quad+C_{\varepsilon}\left(\left|u_{n}\right|^{q-1}+|u|^{q-1}\right)\right]\left|u_{n}-u\right| d x \\
& \leq \varepsilon \int_{\mathbb{R}^{N}}\left(\left|u_{n}\right|^{p}+|u|^{p}+\left|u_{n}\right|^{p-1}|u|\right. \\
& \left.+|u|^{p-1}\left|u_{n}\right|\right) d x+C_{\varepsilon} \int_{\mathbb{R}^{N}}\left(\left|u_{n}\right|^{q-1}\left|u_{n}-u\right|\right.
\end{aligned}
$$

$$
\begin{aligned}
& \left.+|u|^{q-1}\left|u_{n}-u\right|\right) d x \leq \varepsilon\left(\Lambda_{1}+\Lambda_{2}+\Lambda_{3}\right. \\
& \left.+\|u\|_{p}^{p}\right)+2 C_{\varepsilon}\left(\sup _{n}\left\|u_{n}\right\|_{q}^{q-1}+\|u\|_{q}^{q-1}\right)\left\|u_{n}-u\right\|_{q} .
\end{aligned}
$$

Since $\left\{u_{n}\right\}$ is bounded in $L^{q}\left(\mathbb{R}^{N}\right)$ and $\varepsilon$ is arbitrarily small, we have

$$
\int_{\mathbb{R}^{N}}\left(f\left(x, u_{n}\right)-f(x, u)\right)\left(u_{n}-u\right) d x \longrightarrow 0,
$$

$$
\text { as } n \longrightarrow \infty \text {. }
$$

By (62) and

$$
\begin{aligned}
\left\|u_{n}-u\right\|^{p} & \\
= & \int \frac{\left|\left(u_{n}(x)-u_{n}(y)\right)-(u(x)-u(y))\right|^{p}}{|x-y|^{N+\alpha p}} d x d y \\
& \quad+\int V(x)\left|u_{n}(x)-u(x)\right|^{p} d x
\end{aligned}
$$

we have

$$
\begin{aligned}
& \left\langle\Phi_{\lambda}^{\prime}\left(u_{n}\right)-\Phi_{\lambda}^{\prime}(u), u_{n}-u\right\rangle \\
& =\int \frac{\left|u_{n}(x)-u_{n}(y)\right|^{p-2}\left(u_{n}(x)-u_{n}(y)\right)-|u(x)-u(y)|^{p-2}(u(x)-u(y))}{|x-y|^{N+\alpha p}}\left(u_{n}(x)-u_{n}(y)-u(x)+u(y)\right) d x d y \\
& \quad+\lambda \int V(x)\left[\left|u_{n}\right|^{p-2} u_{n}-|u|^{p-2} u\right]\left(u_{n}-u\right) d x-\int\left(f\left(x, u_{n}\right)-f(x, u)\right)\left(u_{n}-u\right) d x \\
& \quad \geq c_{1} \int \frac{\left|\left(u_{n}(x)-u_{n}(y)\right)-(u(x)-u(y))\right|^{p}}{|x-y|^{N+\alpha p}} d x d y+\lambda c_{2} \int V(x)\left|u_{n}-u\right|^{p} d x-o(1) \\
& \geq \min \left\{c_{1}, \lambda c_{2}\right\}\left\|u_{n}-u\right\|^{p}-o(1)
\end{aligned}
$$

where we have used the following elementary inequality:

$$
\left(|a|^{p-2} a-|b|^{p-2} b\right)(a-b) \geq c|a-b|^{p},
$$

where the constant $c$ is independent from the variable $a$ and $b$. Recall that $u_{n} \rightarrow u, \Phi_{\lambda}^{\prime}\left(u_{n}\right) \rightarrow 0$ as $n \rightarrow \infty$; it is clear that

$$
\left\langle\Phi_{\lambda}^{\prime}\left(u_{n}\right)-\Phi_{\lambda}^{\prime}(u), u_{n}-u\right\rangle \longrightarrow 0, \quad \text { as } n \longrightarrow \infty
$$

From (64), (66), we have $\left\|u_{n}-u\right\| \rightarrow 0$ as $n \rightarrow \infty$. Therefore, $\Phi_{\lambda}(u)$ satisfies Cerami condition.

Since Lemmas 5-8 hold, the Mountain Pass Theorem [28] gives that (5) has a nontrivial solution $u_{\lambda}$ satisfying

$$
\Phi_{\lambda}^{\prime}\left(u_{\lambda}\right)=0
$$

$$
\begin{aligned}
c_{\lambda} & =\Phi_{\lambda}\left(u_{\lambda}\right)=\inf _{\gamma \in \Gamma} \max _{t \in[0,1]} \Phi_{\lambda}(\gamma(t))>\Phi_{\lambda}(0) \\
& =0,
\end{aligned}
$$

where $\Gamma=\left\{\Upsilon \in C\left([0,1], X^{\alpha}\right) \mid \Phi_{\lambda}(0)=0, \Phi_{\lambda}(1)=e\right\}$ and $e$ from Lemma 6.

Lemma 9. Let $\lambda_{k} \rightarrow 0$ and $\left\{u_{k}\right\} \subset E$ be a sequence of critical points of $\Phi_{\lambda_{k}}$ satisfying $\Phi_{\lambda_{k}}^{\prime}\left(u_{k}\right)=0$ and $\Phi_{\lambda_{k}}\left(u_{k}\right) \leq C$ for some $C$ independent of $k$. Then, up to a subsequence $u_{k} \rightarrow u$ in $X^{\alpha}$ as $k \rightarrow \infty$ and $u$ is a critical point of $\Phi$.

Proof. We first claim that $\left\{u_{k}\right\}$ is bounded in $X^{\alpha}$. Observe that

$$
c_{\lambda_{k}}=\Phi_{\lambda_{k}}\left(u_{k}\right)-\frac{1}{p} \Phi_{\lambda_{k}}^{\prime}\left(u_{k}\right) u_{k}=\int_{\mathbb{R}^{N}} \widetilde{F}\left(x, u_{k}\right) d x
$$


Arguing indirectly, assume by contradiction that $\left\|u_{k}\right\| \rightarrow \infty$; then $\left\|u_{k}\right\|_{\lambda_{k}, p} \rightarrow \infty$. Set $v_{k}=u_{k} /\left\|u_{k}\right\|_{\lambda_{k}, p}$; then $\left\|v_{k}\right\|_{\lambda_{k}, p}=1$. By Lemma 3, one has $\left\|v_{k}\right\|_{s} \leq \gamma_{s}\left\|v_{k}\right\|_{\lambda, p}=\gamma_{s}$ for $s \in\left[p, p_{\alpha}^{*}\right)$. Observe that

$$
\begin{aligned}
\Phi_{\lambda_{k}}^{\prime}\left(u_{k}\right) u_{k} & =\left\|u_{k}\right\|_{\lambda_{k}, p}^{p}\left(1-\int_{\mathbb{R}^{N}} \frac{f\left(x, u_{k}\right) v_{k}}{\left\|u_{k}\right\|_{\lambda_{k}, p}^{p-1}} d x\right) \\
& =0
\end{aligned}
$$

it follows that

$$
\int_{\mathbb{R}^{N}} \frac{f\left(x, u_{k}\right) v_{k}}{\left\|u_{k}\right\|_{\lambda_{k}, p}^{p-1}} d x=\int_{\mathbb{R}^{N}} \frac{f\left(x, u_{k}\right)}{u_{k}^{p-1}} v_{k}^{p} d x=1 .
$$

Set for $r \geq 0$

$$
h(r):=\inf \left\{\widetilde{F}(x, u) \mid x \in \mathbb{R}^{N}, u \in \mathbb{R} \text { with }|u| \geq r\right\} .
$$

By $\left(f_{3}\right), h(r)>0$ for all $r>0$, and $h(r) \rightarrow+\infty$ as $r \rightarrow+\infty$. For $0 \leq a<b$ let

$$
\begin{aligned}
& \Omega_{k}(a, b)=\left\{x \in \mathbb{R}^{N}|a \leq| u_{k}(x) \mid<b\right\} \\
& c_{a}^{b}:=\inf \left\{\frac{\widetilde{F}(x, u)}{u^{p}} \mid x \in \mathbb{R}^{N}, u \in \mathbb{R} \text { with } a \leq|u|\right. \\
& \quad \leq b\} .
\end{aligned}
$$

Since $\widetilde{F}(x, u)>0$ if $u \neq 0$, one has $c_{a}^{b}>0$ and

$$
\tilde{F}\left(x, u_{k}(x)\right) \geq c_{a}^{b}\left|u_{k}(x)\right|^{p}, \quad \forall x \in \Omega_{k}(a, b) .
$$

It follows from (68) that

$$
\begin{aligned}
c_{\lambda_{k}}= & \int_{\Omega_{k}(0, a)} \tilde{F}\left(x, u_{k}\right) d x+\int_{\Omega_{k}(a, b)} \tilde{F}\left(x, u_{k}\right) d x \\
& +\int_{\Omega_{k}(b, \infty)} \widetilde{F}\left(x, u_{k}\right) d x \\
\geq & \int_{\Omega_{k}(0, a)} \widetilde{F}\left(x, u_{k}\right) d x+c_{a}^{b} \int_{\Omega_{k}(a, b)}\left|u_{k}\right|^{p} d x \\
& +h(b)\left|\Omega_{k}(b,+\infty)\right| .
\end{aligned}
$$

Invoking $\left(f_{3}\right)$, set $\tau:=p \sigma /(\sigma-1)$ and $\sigma^{*}=\sigma /(\sigma-1)=\tau / p$. Since $\sigma>\max \{1, N / p \alpha\}$ one sees $\tau \in\left(p, p_{\alpha}^{*}\right)$. Fix arbitrarily $s \in\left(\tau, p_{\alpha}^{*}\right)$. Using (74),

$$
\left|\Omega_{k}(b,+\infty)\right| \leq \frac{c_{\lambda_{k}}}{h(b)} \longrightarrow 0
$$

as $b \rightarrow+\infty$ uniformly in $k$, which implies by Hölder inequality that

$$
\begin{aligned}
& \int_{\Omega_{k}(b,+\infty)}\left|v_{k}\right|^{\tau} d x \\
& \quad \leq\left[\int_{\Omega_{k}(b,+\infty)}\left|v_{k}\right|^{s} d x\right]^{\tau / s}\left|\Omega_{k}(b,+\infty)\right|^{1-\tau / s} \\
& \leq \gamma_{s}^{\tau}\left|\Omega_{k}(b,+\infty)\right|^{1-\tau / s} \longrightarrow 0
\end{aligned}
$$

as $b \rightarrow+\infty$ uniformly in $k$. Using (74) again, for any fix $0<$ $a<b$,

$$
\begin{aligned}
\int_{\Omega_{k}(a, b)}\left|v_{k}\right|^{p} d x & =\frac{1}{\left\|u_{k}\right\|_{\lambda_{k}, p}^{p}} \int_{\Omega_{k}(a, b)}\left|u_{k}\right|^{p} d x \\
& \leq \frac{c_{\lambda_{k}}}{c_{a}^{b}\left\|u_{k}\right\|_{\lambda_{k}, p}^{p}} \longrightarrow 0
\end{aligned}
$$

as $k \rightarrow+\infty$.

Let $0<\varepsilon<1 / 3$. By $\left(f_{2}\right)$ there is $a_{\varepsilon}>0$ such that $|f(x, u)|<\left(\varepsilon / \gamma_{p}\right)|u|^{p-1}$ for all $|u| \leq a_{\varepsilon}$; consequently,

$$
\begin{aligned}
& \int_{\Omega_{k}\left(0, a_{\varepsilon}\right)} \frac{f\left(x, u_{k}\right)}{u_{k}^{p-1}} v_{k}^{p} d x \leq \int_{\Omega_{k}\left(0, a_{\varepsilon}\right)} \frac{\varepsilon}{\gamma_{p}}\left|v_{k}\right|^{p} d x \\
& \leq \frac{\varepsilon}{\gamma_{p}}\left\|v_{k}\right\|_{p}^{p} \leq \varepsilon
\end{aligned}
$$

for all $k$. Obviously, by (31), there exists a constant $\widetilde{C}(k)>0$ such that

$$
\begin{aligned}
\int_{\mathbb{R}^{N}} c_{0} \widetilde{F}\left(x, u_{k}\right) d x \leq & \frac{2 c_{0} \epsilon}{p} \int_{\mathbb{R}^{N}}\left|u_{k}\right|^{p} d x \\
& +c_{0}\left(\frac{1}{p}+\frac{1}{q}\right) C_{\epsilon} \int_{\mathbb{R}^{N}}\left|u_{k}\right|^{q} d x \\
\leq & \frac{2 c_{0} \epsilon \gamma_{p}^{p}}{p}\left\|u_{k}\right\|^{p} \\
& +c_{0}\left(\frac{1}{p}+\frac{1}{q}\right) \gamma_{q}^{q}\left\|u_{k}\right\|^{q}<\widetilde{C}(k)
\end{aligned}
$$

for all $u_{k} \in X^{\alpha}$. By $\left(f_{3}\right),(68),(79)$, and Hölder inequality we can take $b_{\varepsilon} \geq r_{0}$ large so that

$$
\begin{aligned}
& \int_{\Omega_{k}\left(b_{\varepsilon},+\infty\right)} \frac{f\left(x, u_{k}\right)}{u_{k}^{p-1}} v_{k}^{p} d x \\
& \leq\left(\int_{\Omega_{k}\left(b_{\varepsilon},+\infty\right)} \frac{\left|f\left(x, u_{k}\right)\right|^{\sigma}}{\left|u_{k}\right|^{\sigma(p-1)}} d x\right)^{1 / \sigma} \\
& \cdot\left(\int_{\Omega_{k}\left(b_{\varepsilon},+\infty\right)}\left|v_{k}\right|^{\tau} d x\right)^{1 / \sigma^{*}} \\
& \leq\left(\int_{\mathbb{R}^{N}} c_{0} \widetilde{F}\left(x, u_{k}\right) d x\right)^{1 / \sigma}\left(\int_{\mathbb{R}^{N}}\left|v_{k}\right|^{\tau} d x\right)^{(p-1) / \tau} \\
& \quad\left(\int_{\Omega_{k}\left(b_{\varepsilon},+\infty\right)}\left|v_{k}\right|^{\tau} d x\right)^{1 / \tau}<\varepsilon
\end{aligned}
$$

for all $k$. Note that there is $\gamma=\gamma(\varepsilon)>0$ independent of $k$ such that $\left|f\left(x, u_{k}\right)\right| \leq \gamma\left|u_{k}\right|^{p-1}$ for $x \in \Omega_{k}\left(a_{\varepsilon}, b_{\varepsilon}\right)$. By (77) there is $k_{0}$ such that

$$
\int_{\Omega_{k}\left(a_{\varepsilon}, b_{\varepsilon}\right)} \frac{f\left(x, u_{k}\right)}{u_{k}^{p-1}} v_{k}^{p} d x \leq \gamma \int_{\Omega_{k}\left(a_{\varepsilon}, b_{\varepsilon}\right)}\left|v_{k}\right|^{p} d x<\varepsilon
$$


for all $k \geq k_{0}$. Now the combination of (78), (80), and (81) implies that for $k \geq k_{0}$

$$
\int_{\mathbb{R}^{N}} \frac{f\left(x, u_{k}\right)}{u_{k}^{p-1}} v_{k}^{p} d x<3 \varepsilon<1
$$

which contradicts with (70). Hence $\left\{u_{k}\right\}$ is bounded in $X^{\alpha}$. We may assume up to a subsequence $\left\{u_{k}\right\}$ converges to $u$ weakly in $X^{\alpha}$. By Hölder inequality, we have

$$
\begin{aligned}
& \lambda_{k} \int_{\mathbb{R}^{N}} V(x)\left|u_{k}\right|^{p-2} u_{k} v d x \\
& \quad \leq \lambda_{k} \int_{\mathbb{R}^{N}}[V(x)]^{(p-1) / p}\left|u_{k}\right|^{p-1}[V(x)]^{1 / p} v d x \\
& \quad \leq \lambda_{k}\left[\int_{\mathbb{R}^{N}} V(x)\left|u_{k}\right|^{p} d x\right]^{(p-1) / p} \\
& \quad \cdot\left[\int_{\mathbb{R}^{N}} V(x)|v|^{p} d x\right]^{1 / p} \longrightarrow 0, \text { as } k \longrightarrow \infty .
\end{aligned}
$$

By the weakly continuity of $\Phi^{\prime}$ and (83), we have

$$
\begin{aligned}
& \left\langle\Phi^{\prime}(u), v\right\rangle=\lim _{k \rightarrow \infty}\left\langle\Phi^{\prime}\left(u_{k}\right), v\right\rangle \\
& =\lim _{k \rightarrow \infty}\left[\left\langle\Phi_{\lambda_{k}}^{\prime}\left(u_{k}\right), v\right\rangle\right. \\
& \left.-\lambda_{k} \int_{\mathbb{R}^{N}} V(x)\left|u_{k}\right|^{p-2} u_{k} v d x\right] \\
& =-\lim _{k \rightarrow \infty} \lambda_{k} \int_{\mathbb{R}^{N}} V(x)\left|u_{k}\right|^{p-2} u_{k} v d x=0
\end{aligned}
$$

for any $v \in X^{\alpha}$. Hence, $u$ is critical point of $\Phi(u)$.

Proof of Theorem 1. By Lemma 6, there exists a constant $C>$ 0 , independent of $\lambda$, such that

$$
c_{\lambda}=\inf _{\gamma \in \Gamma} \max _{t \in[0,1]} \Phi_{\lambda}(\gamma(t)) \leq \sup _{t \geq 0} \Phi_{1}(t T u) \leq C .
$$

Then, we can choose a sequence $\lambda_{k} \rightarrow 0$. Assume that $\left\{u_{k}\right\} \subset$ $W^{\alpha, p}$ is a sequence of critical points of $\Phi_{\lambda_{k}}$. According to Lemma $9, u$ is a critical point of $\Phi(u)$ on $W^{\alpha, p}$; it is suffice to show that $u \neq 0$. Indeed, if $u$ is vanishing, then

$$
\delta:=\lim _{k \rightarrow+\infty} \sup _{y \in R^{N}} \int_{B_{1}(y)}\left|u_{k}(x)\right|^{p} d x=0 .
$$

By Lemma 4, we have

$$
u_{k} \longrightarrow 0 \text { in } L^{q}\left(R^{N}\right) \text {, for } p<q<p_{\alpha}^{*} .
$$

By $\left(f_{1}\right),\left(f_{2}\right)$, for $\varepsilon>0$, there exists $C_{\varepsilon}>0$ such that

$$
\begin{aligned}
|f(x, u) u| & \leq \varepsilon|u|^{p}+C_{\varepsilon}|u|^{q} \\
0 & =\left\langle\Phi_{\lambda_{k}}^{\prime}\left(u_{k}\right), u_{k}\right\rangle \\
& =\left\|u_{k}\right\|_{\alpha, p}^{p}+\lambda_{k} \int_{\mathbb{R}^{N}} V(x) u_{k}^{p} d x
\end{aligned}
$$

$$
\begin{gathered}
-\int_{\mathbb{R}^{N}} f\left(x, u_{k}\right) u_{k} d x \\
\geq\left\|u_{k}\right\|_{\alpha, p}^{p}-\varepsilon\left\|u_{k}\right\|_{p}^{p}-C_{\varepsilon}\left\|u_{k}\right\|_{q}^{q} \\
\geq C\left\|u_{k}\right\|_{q}^{p}-C_{\varepsilon}\left\|u_{k}\right\|_{q}^{q}
\end{gathered}
$$

since $\varepsilon$ is arbitrary small. Hence, we have $\left\|u_{k}\right\|_{q} \geq[C /$ $\left.C_{\varepsilon}\right]^{1 /(q-p)}>0$. This conflicts with (87); thus $u$ is nonvanishing, $\delta>0$. Therefore, by standard method, we can obtain $u \neq 0$. The proof is completed.

\section{Conflicts of Interest}

The authors declare that they have no conflicts of interest.

\section{Acknowledgments}

This work is supported by the National Natural Science Foundation of China (Grants no. 11461016 and no. 11571370), Hainan Natural Science Foundation (Grant no. 117005), China Postdoctoral Science Foundation funded project (Grant no. 2017M612577), and Young Foundation of Hainan University (Grant no. hdkyxj201718).

\section{References}

[1] D. Applebaum, "Lévy processes-from probability to finance and quantum groups," Notices of the American Mathematical Society, vol. 51, no. 11, pp. 1336-1347, 2004.

[2] N. Laskin, "Fractional quantum mechanics and Lévy path integrals," Physics Letters A, vol. 268, no. 4-6, pp. 298-305, 2000.

[3] R. Metzler and J. Klafter, "The restaurant at the end of the random walk: recent developments in the description of anomalous transport by fractional dynamics," Journal of Physics A: Mathematical and General, vol. 37, no. 31, pp. R161-R208, 2004.

[4] L. Caffarelli and L. Silvestre, "An extension problem related to the fractional Laplacian," Communications in Partial Differential Equations, vol. 32, no. 7-9, pp. 1245-1260, 2007.

[5] P. Pucci, M. Xiang, and B. Zhang, "Multiple solutions for nonhomogeneous Schrodinger-Kirchhoff type equations involving the fractional $p$-Laplacian in RN," Calculus of Variations and Partial Differential Equations, vol. 54, no. 3, pp. 2785-2806, 2015.

[6] M. Xiang, B. Zhang, and V. t. Radulescu, "Existence of solutions for perturbed fractional p-Laplacian equations," Journal of Differential Equations, vol. 260, no. 2, pp. 1392-1413, 2016.

[7] M. Xiang, B. Zhang, and V. t. Radulescu, "Multiplicity of solutions for a class of quasilinear Kirchhoff system involving the fractional $p$-Laplacian Nonlinearity," Nonlinearity, vol. 29, no. 10, pp. 3186-3205, 2016.

[8] J. Zhang, X. Tang, and W. Zhang, "Existence of infinitely many solutions for a quasilinear elliptic equation," Applied Mathematics Letters, vol. 37, pp. 131-135, 2014.

[9] J. Zhang, X. Tang, and W. Zhang, "Existence of multiple solutions of Kirchhoff type equation with sign-changing potential," Applied Mathematics and Computation, vol. 242, pp. 491-499, 2014. 
[10] L. Ambrosio, G. De Philippis, and L. Martinazzi, "Gammaconvergence of nonlocal perimeter functionals," Manuscripta Mathematica, vol. 134, no. 3-4, pp. 377-403, 2011.

[11] B. Barrios, E. Colorado, and A. de Pablo, "On some critical problems for the fractional Laplacian operator," Journal of Differential Equations, vol. 252, no. 11, pp. 6133-6162, 2012.

[12] Z. Binlin, G. Molica Bisci, and R. Servadei, "Superlinear nonlocal fractional problems with infinitely many solutions," Nonlinearity, vol. 28, no. 7, pp. 2247-2264, 2015.

[13] L. Caffarelli, J.-M. Roquejoffre, and O. Savin, "Nonlocal minimal surfaces," Communications on Pure and Applied Mathematics, vol. 63, no. 9, pp. 1111-1144, 2010.

[14] P. Felmer, A. Quaas, and J. Tan, "Positive solutions of the nonlinear Schrodinger equation with the fractional Laplacian," Proceedings of the Royal Society of Edinburgh, Section: A Mathematics, vol. 142, no. 6, pp. 1237-1262, 2012.

[15] A. Iannizzotto and M. Squassina, "1/2-Laplacian problems with exponential nonlinearity," Journal of Mathematical Analysis and Applications, vol. 414, no. 1, pp. 372-385, 2014.

[16] G. Molica Bisci, D. s. Repovs, and R. Servadei, "Nontrivial solutions of superlinear nonlocal problems," Forum Mathematicum, vol. 28, no. 6, pp. 1095-1110, 2016.

[17] G. M. Bisci and V.t. Radulescu, "Ground state solutions of scalar field fractional Schroedinger equations," Calculus of Variations and Partial Differential Equations, vol. 54, no. 3, pp. 2985-3008, 2015.

[18] K. Teng, "Two nontrivial solutions for hemivariational inequalities driven by nonlocal elliptic operators," Nonlinear Analysis: Real World Applications, vol. 14, no. 1, pp. 867-874, 2013.

[19] J. Zhang, X. Tang, and W. Zhang, "Semiclassical solutions for a class of Schrödinger system with magnetic potentials," Journal of Mathematical Analysis and Applications, vol. 414, no. 1, pp. 357-371, 2014.

[20] J. Zhang, X. Tang, and W. Zhang, "Ground states for diffusion system with periodic and asymptotically periodic nonlinearity," Computers \& Mathematics with Applications, vol. 71, no. 2, pp. 633-641, 2016.

[21] J. Zhang, X. Tang, and W. Zhang, "Ground state solutions for a class of nonlinear Maxwell-Dirac system," Topological Methods in Nonlinear Analysis, vol. 46, no. 2, pp. 785-798, 2015.

[22] E. Di Nezza, G. Palatucci, and E. Valdinoci, "Hitchhiker's guide to the fractional Sobolev spaces," Bulletin des Sciences Mathématiques, vol. 136, no. 5, pp. 521-573, 2012.

[23] G. Molica Bisci, V. D. Radulescu, and R. Servadei, Variational Methods for Nonlocal Fractional Problems, vol. 162 of Encyclopedia of Mathematics and its Applications, Cambridge University Press, Cambridge, UK, 2016.

[24] D. Qin, X. Tang, and J. Zhang, "Multiple solutions for semilinear elliptic equations with sign-changing potential and nonlinearity," Electronic Journal of Differential Equations, No. 207, 9 pages, 2013.

[25] X. H. Tang, "Infinitely many solutions for semilinear Schrödinger equations with signchanging potential and nonlinearity," Journal of Mathematical Analysis and Applications, vol. 401, no. 1, pp. 407-415, 2013.

[26] W. Zhang, X. Tang, and J. Zhang, "Infinitely many solutions for fourth-order elliptic equations with sign-changing potential," Taiwanese Journal of Mathematics, vol. 18, no. 2, pp. 645-659, 2014.

[27] X. Chang, "Ground state solutions of asymptotically linear fractional Schrödinger equations," Journal of Mathematical Physics, vol. 54, no. 6, Article ID 061504, 2013.
[28] M. Willem, Minimax Theorems, Birkhäuser, Boston, Mass, USA, 1996. 


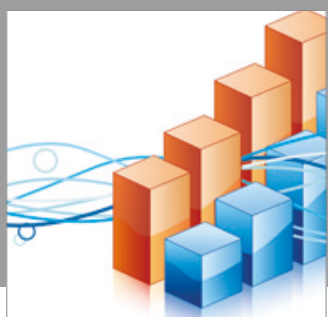

Advances in

Operations Research

vatersals

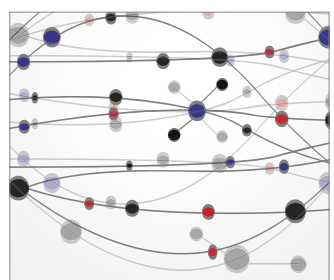

\section{The Scientific} World Journal
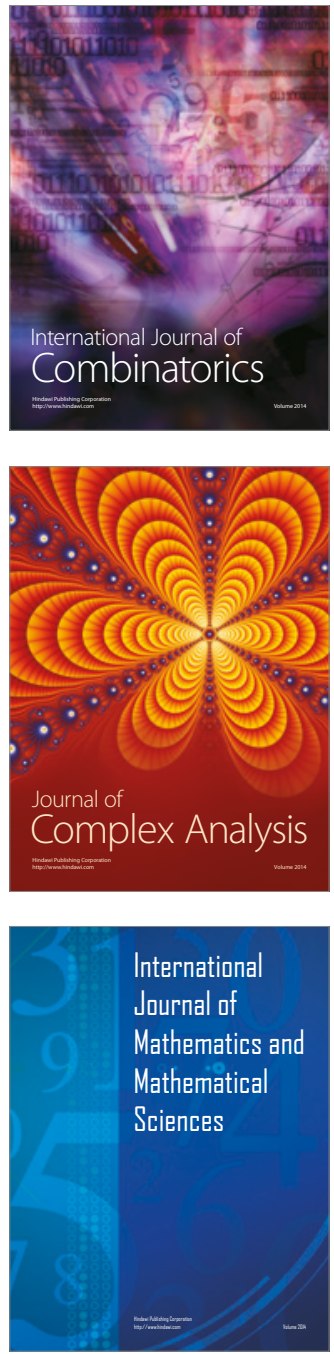
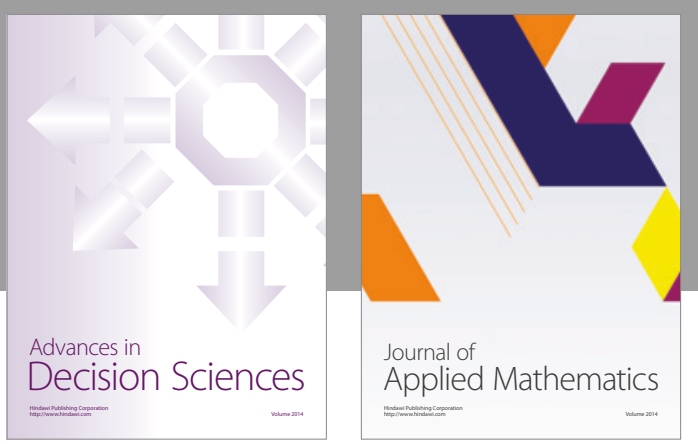

Algebra

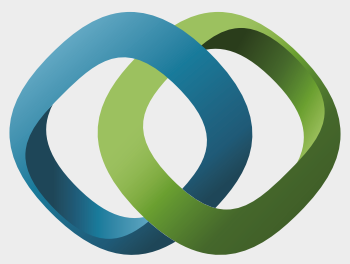

\section{Hindawi}

Submit your manuscripts at

https://www.hindawi.com
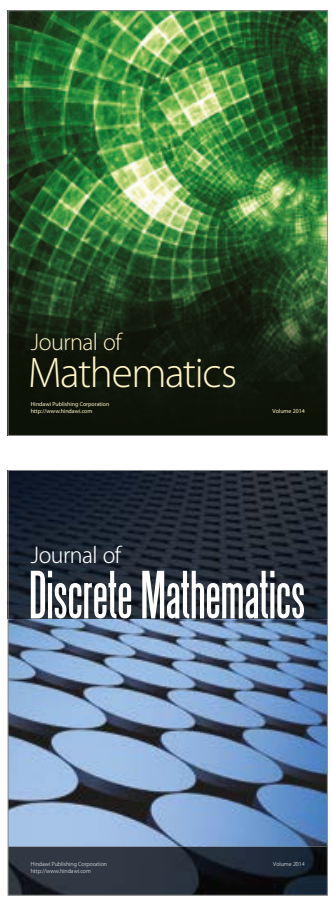

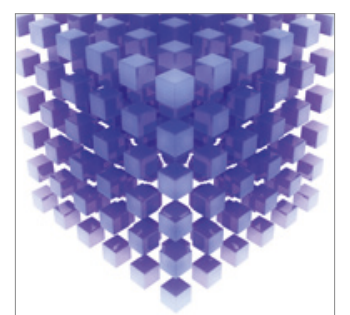

Mathematical Problems in Engineering
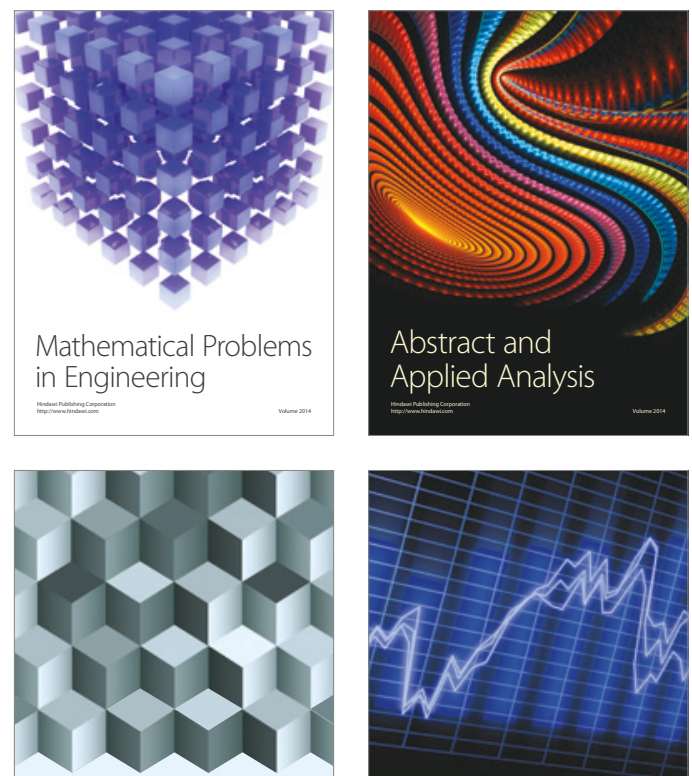

Journal of

Function Spaces

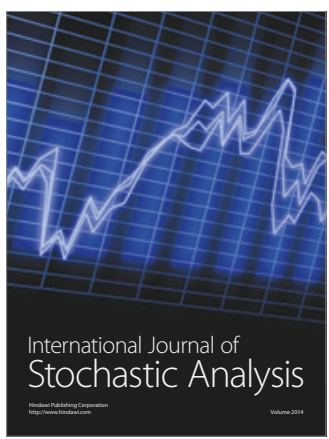

Probability and Statistics
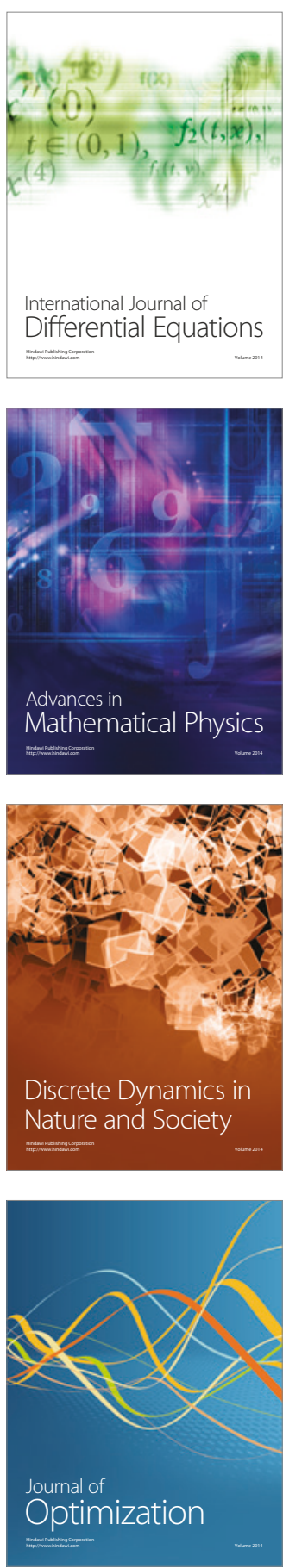